\title{
Diffusion-Limited Unbinding of Small Peptides from PDZ Domains
}

\author{
Fabio Cecconi, ${ }^{\dagger}$ Paolo De Los Rios, $\stackrel{\ddagger}{\ddagger}$ and Francesco Piazza*, \\ INFM-SMC and Instituto dei Sistemi Complessi ISC-CNR, Via dei Taurini 19, 00185 Roma, Italy, and \\ Laboratoire de Biophysique Statistique, EPFL SB ITP, CH-1015, Lausanne, Switzerland
}

Received: April 19, 2007; In Final Form: July 13, 2007

\begin{abstract}
PDZ domains are typical examples of binding motifs mediating the formation of protein-protein assemblies in many different cells. A quantitative characterization of the mechanisms intertwining structure, chemistry, and dynamics with the PDZ function represent a challenge in molecular biology. Here, we investigated the influence of native state topology on the thermodynamics and dissociation kinetics for a PDZ/peptide complex via molecular dynamics simulations based on a coarse-grained description of PDZ domains. Our nativecentric approach neglects chemical details but incorporates the basic structural information to reproduce the protein functional dynamics as it couples to binding. We found that at physiological temperatures the unbinding of a peptide from the PDZ domain becomes increasingly diffusive rather than thermally activated, as a consequence of the significant reduction of the free energy barrier with temperature. In turn, this results in a significant slowing down of the process of 2 orders of magnitude with respect to the conventional Arrhenius extrapolation from low-temperature calculations. Finally, a detailed analysis of a typical unbinding event based on the rupture times of single peptide-PDZ contacts allows us to shed further light on the dissociation mechanism and to elaborate a coherent picture of the relation between function and dynamics in PDZ domains.
\end{abstract}

\section{Introduction}

The role of PDZ domains in the organization of protein complexes at the plasma membrane has been increasingly recognized in the past decade. ${ }^{1}$ Proteins containing several PDZ domains (up to 13 in the MUPP1 protein ${ }^{2}$ ) act as scaffolds that cluster together different transmembrane, membrane associated, and periplasmic proteins involved, among other functions, in signaling pathways ${ }^{3,4}$ and ion permeability. ${ }^{5}$ The participation of PDZ domains in the organization of supramolecular complexes in skeletal muscle cells has also been documented. ${ }^{6,7}$

PDZ domains associate with other proteins by binding their carboxyl-terminal aminoacids, ${ }^{8,9}$ as highlighted by the structure of several PDZ domains, such as the third PDZ domain of PSD95 (postsynaptic density-95/disks large/zonula occludens$1)^{10}$ and the PDZ domain of ZASP (Z-band alternatively spliced PDZ-motif), ${ }^{11}$ although internal structures, such as $\beta$-hairpins, that mimic C-terminal geometries can also be recognized, as in the complex between nNOS (neuronal nitric oxide synthase) and syntrophin. ${ }^{12}$

Recently, due to their central role as key mediators of protein-protein interactions in mammalian cells, PDZ domains have been the object of intense study, with the aim of designing small molecules capable of acting as modulators or inhibitors of the PDZ binding activity in a controlled fashion. Efforts have focused on the design of both nonpeptide ${ }^{13-15}$ and peptide ${ }^{16-18}$ ligands, with the ambition to develop molecular probes to study the biophysical and biochemical properties of PDZ domains and to devise new small-molecule-based therapeutic strategies. Hence, understanding the principles of peptide-PDZ interactions is of great importance.

The geometry and chemistry of binding to PDZ domains involve the fit of the last four to five carboxyl-terminal aminoacids

INFM and Instituto dei Sistemi Complessi ISC-CNR.

$\stackrel{*}{*}$ EPFL SB ITP. into a groove between an $\alpha$-helix and a $\beta$-strand on the PDZ surface (Figure 1), with the last $\mathrm{C}$-terminal residue almost invariably hydrophobic. The specificity of each domain is conferred by a few (two to three) PDZ surface aminoacids that make contacts with the residues in positions -1 to -4 relative to the C-terminal in the target protein. ${ }^{8}$ The surprising simplicity of this binding scheme possibly explains why PDZ domains are one of the most widespread binding modules yet identified, since just a few incremental, concerted mutations involving surface aminoacids (hence unlikely to change the overall protein stability) can tune the affinity of PDZ domains for different targets. On the other hand, a binding architecture that relies on just a few optimized contacts comes at the price of losing strict specificity. Indeed, recent experiments on 26 mouse PDZ domains and domain clusters have confirmed that each PDZ domain can bind to several peptides, ${ }^{19}$ and that each peptide, in turn, can bind to several PDZ domains.

In a recent work, ${ }^{20}$ we have explored via normal mode analysis (NMA) the mechanical aspects of binding of a peptide to a PDZ domain. In line with the results of NMA analysis of several proteins, ${ }^{21-23}$ the picture that emerged was that a limited number of low-frequency modes suffice to reconstruct the observed conformational change from the apo to the complexed structure of the PDZ. The long-range spatial correlations that characterize these modes correspond to a concerted breathing motion of the binding cleft, thus suggesting that functional dynamics is deeply rooted in the native architecture itself. However, despite their success in providing a qualitative picture of the coupling between thermal fluctuations of the structure and the binding deformation, the predictive power of normal modes is still limited by the harmonic approximation. Hence, in order to investigate the progressive detachment of the peptide from the PDZ structure, one must resort to more general computational studies. 


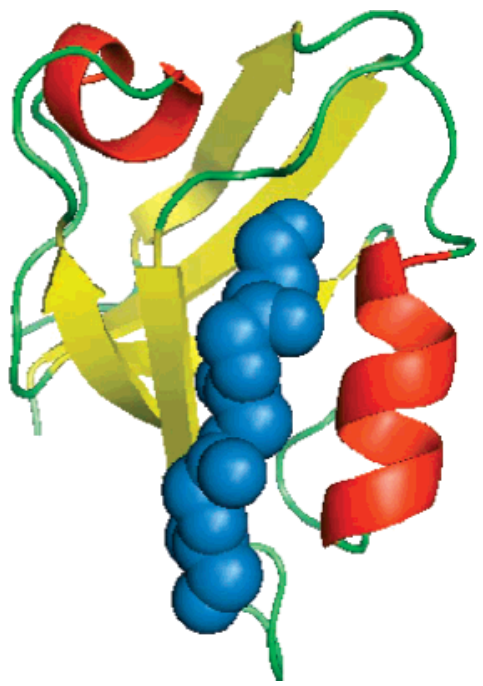

Figure 1. Cartoon representation of the crystal structure of the PDZ3 domain in complex with its target petide (blue). PDB code 1BFE.

Molecular dynamics simulations have played a crucial role in the understanding of the basis of the concomitant selectivity and promiscuity of the PDZ binding dynamics. ${ }^{24,25}$ Basdevant et al., ${ }^{25}$ in particular, have used all-atom molecular dynamics (MD) with a realistic force field to correctly reproduce the experimental ranking, but not the precise values, of binding free energies. The chemical details of the target peptide and of the binding groove have been taken into account, and hydrophobic effects have been shown to be key determinants of the promiscuity, with other interactions conferring stronger or weaker selectivity.

The free energy difference, $\Delta G_{\mathrm{b}}^{\mathrm{u}}$, between the unbound and bound states is customarily related to the dissociation constant, $K_{\mathrm{D}}$, by $K_{\mathrm{D}}=\exp \left(-\Delta G_{\mathrm{b}}^{\mathrm{u}} / k_{\mathrm{B}} T\right)$, where $T$ is the absolute temperature and $k_{\mathrm{B}}$ is the Boltzmann constant. However, the constant $K_{\mathrm{D}}$ is also related to the dynamics of the system by the equation $K_{\mathrm{D}}=k_{\text {off }} / k_{\text {on }}$, where $k_{\text {off }}$ and $k_{\text {on }}$ are the unbinding and binding rates, respectively. At a first approximation, $k_{\text {off }}$ and $k_{\text {on }}$ depend on the free energy difference betweeen the bound and unbound states and the barrier between them. The dissociation constant, $K_{\mathrm{D}}$, therefore, conceals the kinetics of the system, the same value being compatible with both very fast and very slow rates, as long as their ratio does not change. In the case of PDZ domains, dissociation rates range experimentally ${ }^{26,27}$ from a few $s^{-1}$ to $10^{-3} \mathrm{~s}^{-1}$, thus implying that a correct description of the unbinding process would need to capture the PDZ dynamics over comparable time scales. Unfortunately, detailed all-atom MD simulations can at present cover a few tens ${ }^{25}$ and at most reach a hundred nanoseconds. Therefore, in order to observe a typical unbinding event, MD simulations should be from 6 to 10 orders of magnitude longer than currently possible. Hence, one must resort to simpler, coarse-grained models in order to study the kinetics of interaction between a PDZ domain and its target peptide.

Neglecting the atomic detail clearly has both advantages and drawbacks. On the one hand, the reduction in the number of degrees of freedom and the simplified force field of interaction allow longer times scales to be explored, thus making a complete equilibrium description of the binding kinetics possible. On the other hand, the chemical specificities can be reincorporated in the model only at a qualitative level.

In this paper, we employ the Go strategy, ${ }^{28}$ a native-centric scheme, coarse-grained at the residue level to simulate the unbinding dynamics of a peptide from the third PDZ domain of PSD-95 (henceforth referred to as PDZ3). Such a simplified description has already proved successful in the characterization of the role of the native state topology and of its dynamics in protein recognition and binding mechanisms. ${ }^{29,30}$ Our simplified simulation scheme allows us to draw a clear, though approximated, picture of the dissociation kinetics.

The escape of the peptide from the binding groove on the PDZ surface takes place over a free energy landscape that strongly depends on the temperature. At physiologically relevant temperatures, less than $10 \%$ lower than the unfolding temperature (measured to be about $320 \mathrm{~K}$ for the second PDZ domain of PTP-BL $\left.{ }^{31}\right)$, the free energy difference between the bound state and the barrier turns out to be rather small $(0.5 \mathrm{kcal} / \mathrm{mol})$ because of the nearly complete compensation of the enthalpic and entropic components. As a consequence, unbinding at physiological temperatures is eminently a diffusive, rather than thermally activated, process and the unbinding rate is orders of magnitude smaller than an Arrhenius-like extrapolation from low temperatures would suggest.

\section{Methods}

The PDZ3 domain (PDB codes 1BFE and 1BE9 without and with bound peptide, respectively) as resolved by X-ray crystallography ${ }^{10}$ is 110 residues long, but we truncated the chain from Arg309 to Ser393 because the final loop does not participate in the binding mechanism and its large flexibility may hinder some signals specifically involved in the binding.

We adopt the model proposed by Clementi et al. ${ }^{28}$ where successive beads along the chain are connected by stiff harmonic springs, mimicking the peptide bond and maintaining the chain connectivity:

$$
V_{\mathrm{bb}}\left(\vec{r}_{i, i+1}\right)=\frac{1}{2} K \epsilon\left(r_{i, i+1}-R_{i, i+1}\right)^{2}
$$

with a stiffness of $K=1000 / d_{0}^{2}$, where $d_{0}=3.8 \AA$ is the mean equilibrium distance of two consecutive residues along the chain, and $\epsilon=0.84 \mathrm{kcal} / \mathrm{mol}$ (see Results) sets the energy scale. Here, $r_{i j}$ and $R_{i j}$ indicate the distance between residues $i$ and $j$ in a generic conformation and in the native structure (1BFE and 1BE9), respectively.

In line with native-centric schemes, nonbonded (nb) interactions between nonconsecutive $\alpha$-carbons are modeled with Lennard-Jones $12-10$ potentials if the atoms are in contact in the native state according to a given interaction cutoff, $R_{\mathrm{c}}$, and with purely repulsive interactions otherwise

$$
V_{\mathrm{nb}}\left(\vec{r}_{i j}\right)= \begin{cases}\epsilon\left[5\left(\frac{R_{i j}}{r_{i j}}\right)^{12}-6\left(\frac{R_{i j}}{r_{i j}}\right)^{10}\right] & R_{i j} \leq R_{\mathrm{c}} \\ \frac{10 \epsilon}{3}\left(\frac{\sigma}{r_{i j}}\right)^{12} & R_{i j}>R_{\mathrm{c}}\end{cases}
$$

The parameters of the nonbonded interactions are fixed as $R_{\mathrm{c}}$ $=6.5 \AA$ and $\sigma=4.5 \AA$. The force field is completed by the angular interactions

$$
\begin{array}{r}
V_{\text {ang }}=\sum_{i=2}^{N-1} \frac{1}{2} k_{\theta}\left(\theta_{i}-\theta_{i}^{0}\right)^{2}+\sum_{i=3}^{N-2} k_{\phi}^{(1)}\left[1-\cos \left(\phi_{i}-\phi_{i}^{0}\right)\right]+ \\
k_{\phi}^{(3)}\left[1-\cos 3\left(\phi_{i}-\phi_{i}^{0}\right)\right]
\end{array}
$$

where $\theta_{i}$ is the bending angle identified by the three consecutive $\mathrm{C}_{\alpha}$ 's $i-1, i$, and $i+1$ and $\phi_{i}$ is the dihedral angle defined by the two adjacent planes formed by four consecutive $\mathrm{C}_{\alpha}$ 's at $i-$ 
$2, i-1, i$, and $i+1$. The superscript 0 identifies quantities referring to the native conformation. The force field parameters are proportional to the energy scale, $\epsilon$, so that $k_{\theta}=20 \epsilon, k_{\phi}^{(1)}=$ $\epsilon, k_{\phi}^{(3)}=0.5 \epsilon$, and one time unit corresponds to about $3 \mathrm{ps}$, when considering an average aminoacid mass of $110 \mathrm{Da}$.

We have performed fixed-temperature molecular dynamics simulations within the isokinetic scheme, ${ }^{32}$ which provides a correct sampling of the configuration space. We have then applied the multiple histogram technique ${ }^{33}$ to estimate thermodynamic observables such as the internal energy, the specific heat

$$
C_{v}=\frac{\left\langle V^{2}\right\rangle-\langle V\rangle^{2}}{T^{2}}
$$

and the structural similarity, $Q$, of a given conformation with the native structure. The latter parameter is defined as

$$
Q=\frac{\sum_{i>j} \Theta\left(R_{\mathrm{c}}-R_{i j}\right) \Theta\left(R_{\mathrm{c}}-r_{i j}\right)}{\sum_{i>j} \Theta\left(R_{\mathrm{c}}-R_{i j}\right)}
$$

with $\Theta(u)$ being the unitary step function. $Q$ represents the fraction of native contacts present in a given conformation. The multiple histogram technique allows one to construct the free energy profiles $G(Q)=-k_{\mathrm{B}} T \log P(Q)$ as functions of $Q$, which plays the role of a reaction coordinate.

In order to obtain the free energy profiles of unbinding, we employed the umbrella sampling technique. ${ }^{34}$ In particular, we restrained the distance between the peptide and the PDZ3 centers of mass, $\rho=\left|\vec{R}_{\text {pdz }}-\vec{R}_{\text {pept }}\right|$, to a given range of values, $D_{k}(k=$ $\left.1,2, \ldots, N_{\text {samp }}\right)$, via the harmonic umbrella potential

$$
V_{\mathrm{u}}(\rho)=\frac{K_{q}}{2}\left(D_{k}-\rho\right)^{2}
$$

with $K_{q}=0.84 \mathrm{kcal} / \mathrm{mol} / \AA^{2}$. The multiple histogram technique allows then a debiasing at a given temperature by matching all of the histograms for the center-mass distance collected around the different sampling values. By varying the reference temperature, we can obtain different free energy curves.

The statistics of the unbinding times have been collected through Langevin dynamics. The unbinding time is defined here as the time at which the bond distances of all 13 contacts linking the peptide to the PDZ3 exceed a given threshold for the first time. We fixed such thresholds at 1.5 times the corresponding values in the native conformation. However, small variations of this threshold did not result in major changes of the exit time statistics.

\section{Results}

The energy scale, $\epsilon$, can be fixed by comparing the folding temperature, $T_{\mathrm{f}}$, of the model, in units of $\epsilon / k_{\mathrm{B}}$, with experiments. For this purpose, we studied the PDZ domain specific heat, $C_{v}$, as a function of temperature. The thermogram in Figure 2 allows one to identify the unfolding transition at $T_{\mathrm{f}} \approx 0.76$. From available data, ${ }^{31}$ the folding temperature of PDZ3 is close to $323 \mathrm{~K}\left(50^{\circ} \mathrm{C}\right)$, which allows one to set the model energy scale to the value $\epsilon=0.84 \mathrm{kcal} / \mathrm{mol}$. The free energy profile as a function of the fraction of native contacts, $Q$ (Figure 2, inset), is typical of clean two-state transitions, in agreement with experiments, ${ }^{31}$ where folding was found to be affected at most by a high-energy poorly populated intermediate.

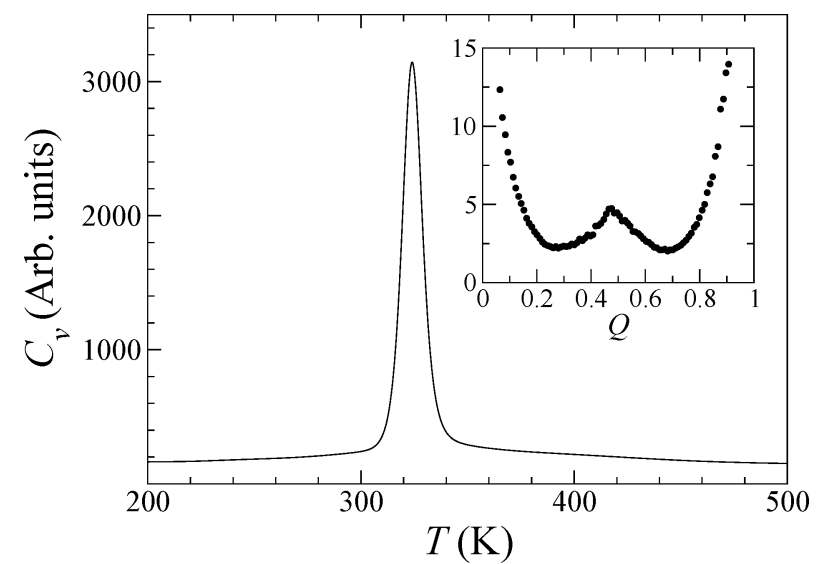

Figure 2. Specific heat curve of Gō-model PDZ domain folding/ unfolding. The inset shows the double well feature of the free energy profile $(\mathrm{kcal} / \mathrm{mol})$ as a function of the fraction of native contacts, $Q$, at the folding temperature.

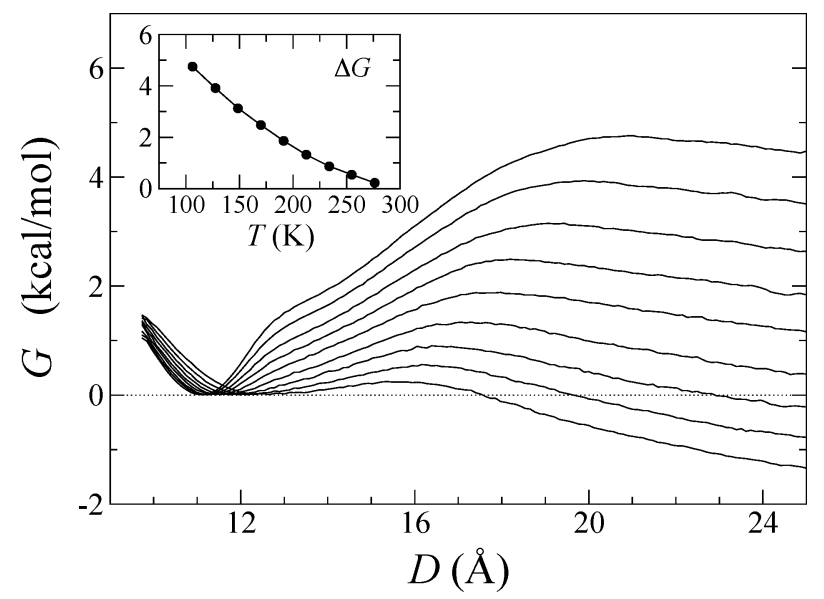

Figure 3. Free energy profile of unbinding along the reaction coordinate at different temperatures below the unfolding of the PDZ3/peptide complex. From top to bottom, the curves refer to temperatures from $T$ $=85 \mathrm{~K}$ up to $T=255 \mathrm{~K}$ in steps of $\Delta T=21 \mathrm{~K}$. The inset shows the temperature dependence of the free energy barrier $(\mathrm{kcal} / \mathrm{mol})$.

A further check of the chosen energy scale may be obtained from a comparison with the experimental stability $\Delta G_{0}=6-6.5$ $\mathrm{kcal} / \mathrm{mol}$ of PDZ3 at $298 \mathrm{~K} .{ }^{31}$ From our simulations, we can estimate $G_{0}$ as the energy difference between the minima in the native and denaturated basins. At $T=298 \mathrm{~K}$, we find $\Delta G_{0}$ $=5 \mathrm{kcal} / \mathrm{mol}$, in good agreement with experiments.

It is our aim to investigate the unbinding dynamics of a small peptide from the PDZ3 domain. Hence, both the unbinding and unfolding transitions have to be carefully located in temperature. The free energy profiles as a function of the distance between the centers of mass of the protein and of the peptide, chosen as the reaction coordinate for the unbinding, are reported in Figure 3 for several temperatures lower than that of unbinding. At low temperatures, the curves are characterized by a well-defined minimum corresponding to the bound state and by a steep free energy barrier that has to be overcome in order for the complex to thermally dissociate. Remarkably, the barrier turns out to decrease rapidly at increasing temperatures, marking an almost complete compensation of the enthalpic barrier by the increase of conformational entropy. The behavior of the dissociation free energy, $\Delta G(T)=G_{\text {barr }}(T)-G_{\min }(T)$, with the temperature is shown in the inset of Figure 3. As expected, $\Delta G$ is a decreasing function of $T$, vanishing at $T_{\text {diss }}=306 \mathrm{~K}$, which locates the spontaneous unbinding $17^{\circ} \mathrm{C}$ below the unfolding temperature. 


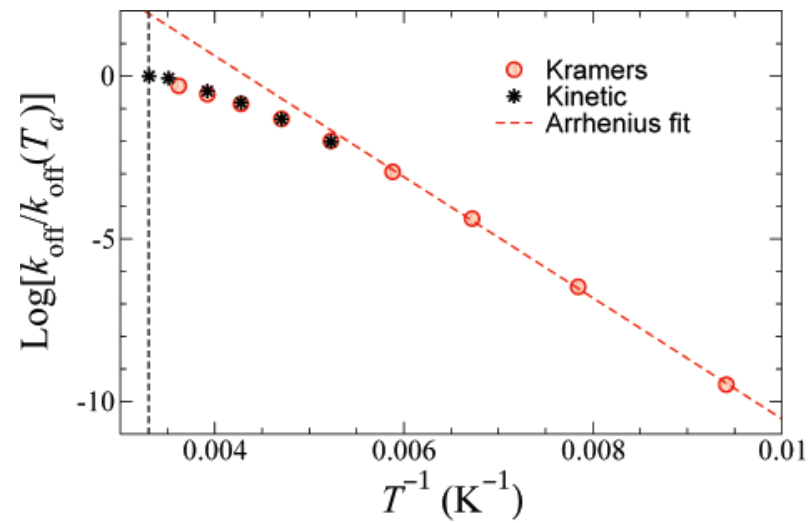

Figure 4. Arrhenius plot comparing the dissociation rates estimated from the free energy profiles of Figure 3 through eq 6 and the rates computed as inverse average times from direct unbinding simulations. Since prefactors are unknown by construction, the latter have been rescaled by an appropriate temperature-independent factor to match the values obtained from Kramers' theory. All rates are normalized by the kinetic rate at room temperature, $T_{a}=300 \mathrm{~K}$. The thick dashed line is the result of an Arrhenius fit with the expression $k_{\text {off }}=a \exp (-\Delta /$ $k_{\mathrm{B}} T$ ), which gives a free energy barrier height of $\Delta \simeq 7 \mathrm{kcal} / \mathrm{mol}$. The vertical dashed line marks the room temperature.

3.1. The Unbinding Dynamics. The data shown in Figure 3 contain the relevant information on the unbinding dynamics. In fact, it is possible to compute the dissociation rates by straight integration of the numerical free energy profiles according to Kramers' theory ${ }^{35}$

$$
k_{\text {off }}(T)=\frac{k_{\mathrm{B}} T}{\gamma \int_{x_{0}}^{x_{\mathrm{b}}} \mathrm{e}^{G(x) / k_{\mathrm{B}} T} \mathrm{~d} x \int_{0}^{x} \mathrm{e}^{-\left(G\left(x^{\prime}\right) / k_{\mathrm{B}} T\right)} \mathrm{d} x^{\prime}}
$$

where $x_{0}$ and $x_{\mathrm{b}}$ are the abscissas of the minimum and of the barrier maximum, respectively. The temperature curve of the dissociation rates as calculated from formula 6 is plotted in Figure 4. Our results clearly show that the simple Arrhenius description $k_{\text {off }} \propto \exp \left(-\Delta G / k_{\mathrm{B}} T\right)$ only holds in the lowtemperature region, where the free energy barrier is high enough to justify the familiar treatment of a thermally activated unbinding. At higher temperatures, the dissociation process slows down considerably with respect to the extrapolated lowtemperature prediction.

This effect is due to the gradual switch from a barrier-limited, thermally activated process at low temperatures to a diffusionlimited process as the unbinding temperature is approached. Indeed, using eq 6 in the limit case where there is a perfectly flat free energy landscape between $x_{0}$ and $x_{\mathrm{b}}$ (and a reflecting wall in $\left.x_{0}\right)$, hence no barrier, $k_{\mathrm{off}}=2\left(k_{\mathrm{B}} T / \gamma\right) /\left(x_{\mathrm{b}}-x_{0}\right)^{2}$, which is the typical rate for particles diffusing from $x_{0}$, with diffusion constant $k_{\mathrm{B}} T / \gamma$, and absorbed at $x_{\mathrm{b}}$. The diffusion-limited $k_{\mathrm{off}}$ value depends only slightly on temperature when represented on an Arrhenius plot (Figure 4).

However, the straightforward application of Kramers' theory at high temperatures may not be legitimate due to the strong reduction of the free energy barriers, that makes the hypotheses of local equilibrium in the free energy minimum and high barrier questionable.

In order to check the validity of the Kramers prediction at high temperatures, we carried out kinetics simulations of unbinding to directly estimate the dissociation rate at a temperature $T$. We observed the time evolution of the PDZ3/peptide complex until a spontaneous unbinding event was recorded. By

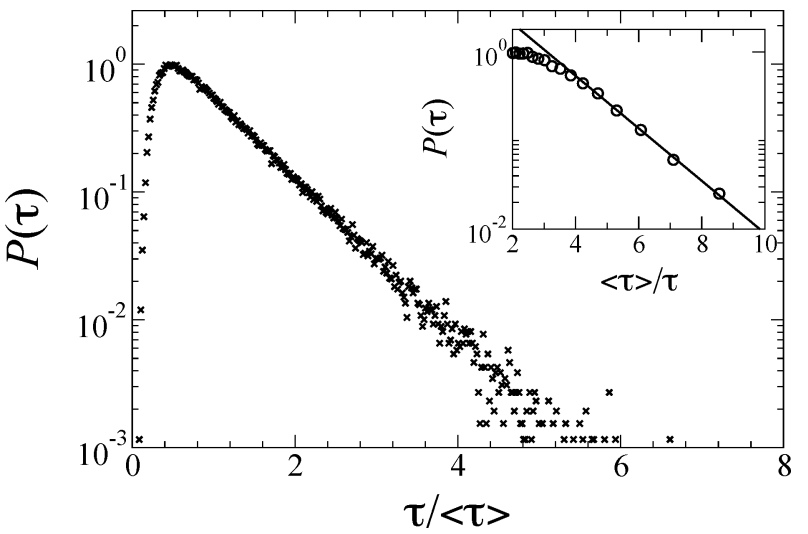

Figure 5. Histogram of the kinetic unbinding times at $T=300 \mathrm{~K}$ (symbols). The solid line in the inset is a fit of the early times region with the first passage time distribution of a diffusive process, $P(\tau)=$ $a \exp \left(-\tau_{0} / \tau\right)$.

doing so, we were able to measure very accurately the lifetimes, $\tau$, of the molecular complex, the temperature-dependent average, $\langle\tau\rangle$, and distributions, $P(\tau)$. The inverse of $\langle\tau\rangle$ provides a measure of the kinetic dissociation rate: $k_{\mathrm{off}}=1 /\langle\tau\rangle$. The results of this procedure are shown in Figure 4 together with the rates estimated from Kramers' formula (eq 6). The agreement between unbinding simulations and the analytical estimates is remarkable at all temperatures where we were able to collect enough statistics for the computation of the average inverse lifetime of the complex. In fact, dissociation events become exponentially rarer at low temperatures, thus requiring unrealistic simulation times in order to collect satisfactory statistics. These results provide a direct confirmation of the validity of eq 6 in the hightemperature region.

That the unbinding process is diffusion-limited at high temperatures is nicely confirmed by the analysis of the histogram of dissociation times. In Figure 5, we show one of such distributions computed at $T=300 \mathrm{~K}$. At long times, $P(\tau)$ decreases exponentially, implying that the slow unbinding events obey the statistics of the waiting times of a Poisson-like process, as should be expected for a simple Arrhenius picture of thermal activation over a barrier. However, it can be clearly appreciated that fast events are under-represented with respect to what the Arrhenius picture would predict. In particular (see inset of Figure 5 ), we find that the left tail of the distribution follows the law $P(\tau) \propto \exp \left(-\tau_{0} / \tau\right)$, which indeed characterizes the distribution of first passage times in a diffusive process.

As a final methodological remark, we wish to comment on using the Langevin scheme for the unbinding simulations. It is known that the kinetics simulated with Langevin MD is strongly dependent on the friction coefficient, $\gamma$. We thus performed different runs at different values of $\gamma$ in order to check the dependence of the unbinding kinetics on the friction. The results of this analysis are reported in Figure 6, where we plot the average peptide dissociation time, $\langle\tau\rangle$, at an intermediate temperature of $T=234 \mathrm{~K}$ as a function of $\gamma$. We explored both the underdamped and overdamped regimes, and compared the numerics with the asymptotic limits of Kramers' theory of barrier crossing ${ }^{36}$

$$
k_{\text {off }} \approx\left\{\begin{array}{l}
\frac{\omega_{0} \mathrm{e}^{-\left(\Delta G / k_{\mathrm{B}} T\right)}}{2 \pi}\left[\frac{\gamma S_{1}}{T}-0.82\left(\frac{\gamma S_{1}}{T}\right)^{3 / 2}\right] \gamma S_{1} \ll T \\
\frac{\omega_{0} \mathrm{e}^{-\left(\Delta G / k_{\mathrm{B}} T\right)}}{2 \pi}\left[\sqrt{\frac{\gamma^{2}}{4 \omega_{\mathrm{b}}^{2}}+1}-\frac{\gamma}{2 \omega_{\mathrm{b}}}\right] \gamma S_{1} \gg T
\end{array}\right.
$$




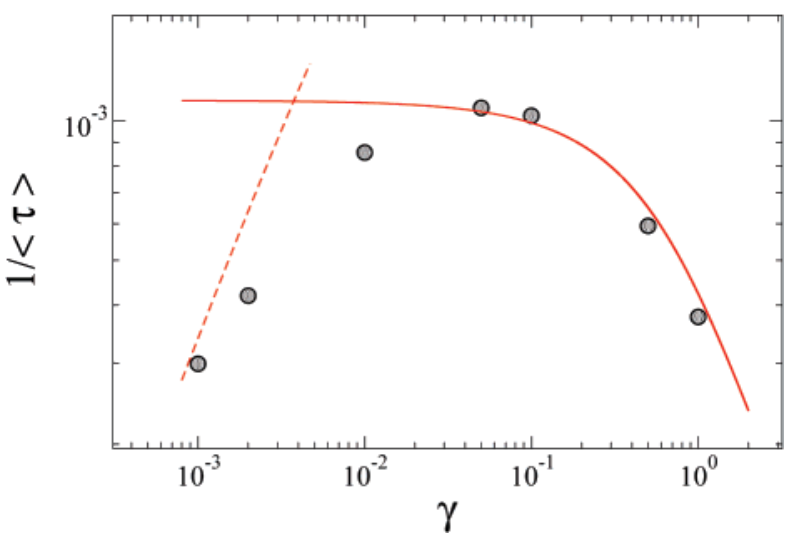

Figure 6. Average kinetic rate at the intermediate temperature $T=$ $234 \mathrm{~K}$ as a function of the friction, $\gamma$, used in the Langevin simulation of the unbinding kinetics. The solid and dashed lines are the predictions of Kramers' theory calculated with the frequencies and free energy barrier estimated from the corresponding simulated free energy profiles of Figure 3. Note that no fitting is performed here.

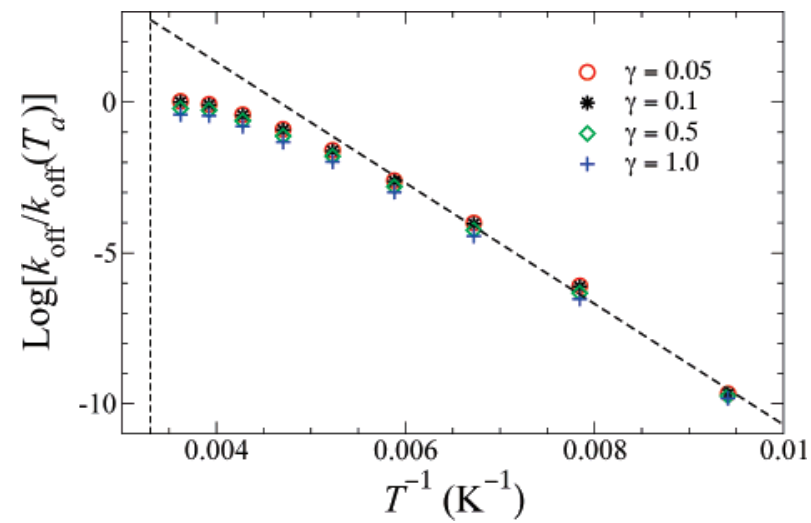

Figure 7. Arrhenius plot comparing the dissociation rates estimated for different values of $\gamma$ from the overdamped formula in eq 7 with the temperature-dependent parameters $\omega_{0}, \omega_{\mathrm{b}}$, and $\Delta G$ estimated from the simulated free energy profiles. All rates are normalized by the kinetic rate at room temperature, $T_{a}=300 \mathrm{~K}$, for $\gamma=0.05$. The vertical dashed line marks the room temperature.

where $\omega_{0}$ and $\omega_{\mathrm{b}}$ are the frequencies at the bottom and saddle points of the free energy profile and

$$
S_{1}=2 \int_{x_{1}}^{x_{\mathrm{b}}} \sqrt{-2 m\left[G(x)-G\left(x_{\mathrm{b}}\right)\right]} \mathrm{d} x
$$

with $x_{1}$ being the left-hand side turning point, i.e., the solution of the equation $G\left(x_{1}\right)=G\left(x_{\mathrm{b}}\right)$ with $x_{1}<x_{0}$. We find that our data indeed converge toward Kramers' estimates in both the underdamped and overdamped regimes, thus showing that the computation of $k_{\text {off }}$ as $1 /\langle\tau\rangle$ can be meaningfully extrapolated to different values of $\gamma$ at different temperatures. In particular, we can make use of the theoretical prediction for the overdamped regime, seen to be valid for values of $\gamma$ approximately greater than 0.1 , for testing whether the results of Figure 4 still hold for other values of the damping parameter when using frequencies and free energy barriers computed numerically. We see from Figure 7 that the essential features of the Arrhenius plot are preserved. Importantly, the room-temperature value of the dissociation rate remains still largely overestimated if extrapolated from the low-temperature values even at larger damping $\gamma$.

The scenario outlined above is consistent with other MD studies employing the Langevin scheme to investigate the kinetics of folding. ${ }^{37}$

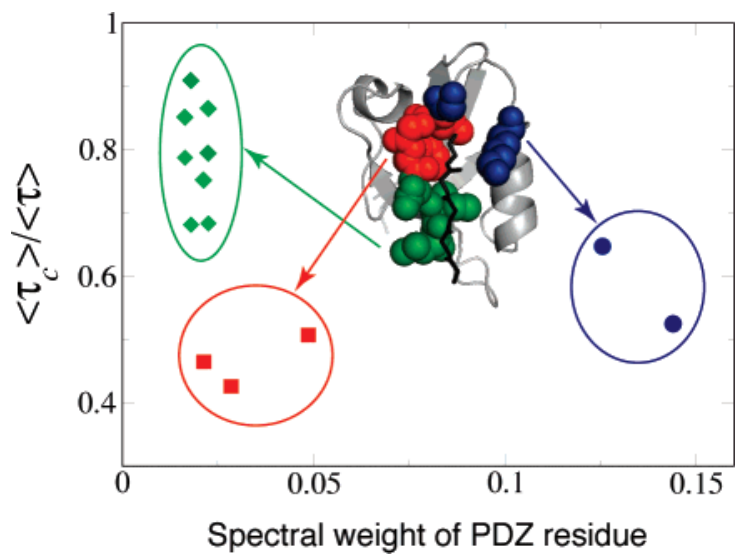

Figure 8. Average breaking times of individual contacts versus the spectral weight on the subset of functional modes of the corresponding residue in the PDZ domain. The breaking times are normalized to the average unbinding time of the peptide as a whole.

3.2. Unbinding Dynamics of Individual Contacts. A more detailed description of the unbinding process can be obtained by considering separately the kinetics of the 13 contacts that keep the peptide bound to the PDZ in the native state. Each of them will be characterized by a different breaking time, $\left\langle\tau_{\mathrm{c}}\right\rangle$, that can be estimated as the average over many dissociation events in a typical unbinding simulation. The ranking beetween such times provides an average picture of the order at which contacts break down during the gradual detachment of the peptide from the PDZ domain.

It is thus interesting to investigate whether a correlation exists between the unbinding event of each PDZ/peptide bond and the dynamics of the residues from the PDZ domain involved in the same link. A relation between the binding dynamics and a specific spatial pattern characterizing a reduced subset of lowfrequency normal modes has been recently suggested by De Los Rios et al. ${ }^{20}$ In the present case, we find four modes among the eigenstates of the Hessian of our force field that match the pattern identified in ref 20 , namely, the set $\mathcal{S}=\{7,8,9,14\}$. We thus introduce an indicator measuring the spectral weight corresponding to that set of modes at each site

$$
w_{i}=\sum_{k \in S} \sum_{\alpha=x, y, z}\left[\xi_{i, \alpha}^{k}\right]^{2}
$$

where $\xi_{i, \alpha}^{k}$ is the $\alpha(\alpha=\{x, y, z\})$ component of the $k$ th normal mode at site $i$. In Figure 8, we plot $\left\langle\tau_{\mathrm{c}}\right\rangle$ versus $w_{i}$ for the 13 binding contacts, where $i$ indicates the residue of the PDZ domain participating in the contact, $\mathrm{c}$, with the peptide.

The data shown are clustered into three sets. The right outermost cluster contains the two contacts that have both a short lifetime and the larger spectral weight. In terms of the latter indicator, these contacts are characterized by the fourth and fifth largest values among all residues. This strongly suggests that functional normal modes are likely to contribute substantially to the loosening of the PDZ/peptide bond and its eventual rupture. The left bottom cluster involves the contacts with small $\left\langle\tau_{\mathrm{c}}\right\rangle$ but whose local fluctuations are poorly captured by the functional modes. Taken together, these first two clusters include the residues forming the hydrophobic pocket: the second group comprises the residues at the bottom of the pocket, whereas the first one includes two residues flanking the entry of the binding pocket. Finally, the last contacts to break up involve the less conserved residues that confer specificity to the different PDZ domains. 
It is interesting to note that our results compare favorably with the finding of evolutionary conserved energetic pathways in PDZ domains as revealed by statistical mechanics measures of multiple structure alignments. ${ }^{38,39}$ In fact, the normal mode patterns corresponding to the modes likely to drive the loosening of the binding pocket tie in with the bioinformatic results, notably revealing a spatial correlation between the motion of the helix flanking the groove and more distant residues (in the neighborhood of positions 21 and 66). ${ }^{20}$

Overall, these results outline the following picture of a typical unbinding event. The first contacts to loosen up are those involving residues from the hydrophobic binding pocket, part of which are involved in the breathing pattern of the cleft as highlighted from normal mode analysis. This observation provides a nice example of the interpretation of a specific normal mode pattern as a precursory feature of a much larger amplitude motion. The contacts that break up the last are those from the surface region of the binding pocket, the ones associated with binding specificity and thus the less conserved ones. Since these residues are obviously more important as far as their chemical details are concerned, it is not surprising that a dynamical investigation based on a purely topological model does not assign them a key role in the unbinding kinetics.

\section{Conclusions and Discussion}

The central result of our analysis of the unbinding of peptides from the PDZ3 domain is that the bound state is stabilized over a physiologically relevant temperature interval.

Counterintuitively, this happens as a direct consequence of the strong temperature-dependent quenching of the free energy barrier in the proximity of the functional region of temperatures. As a matter of fact, such a slowdown of the dissociation reflects the gradual change in nature of the fastest events from thermally activated to diffusive. Indeed, as the free energy barrier gets substantially reduced at increasing temperatures due to the entropy-enthalpy compensation typical in the proximity of the unfolding transition, Kramers' formula predicts a diffusion dominated unbinding process, and kinetic simulations confirm such a picture. Overall, diffusion slows down the process of 2 orders of magnitude with respect to the prediction of the Arrhenius formula extrapolated from the low-temperature region.

The spectral analysis of the breaking time of the contacts involved in the binding helps shed further light on the unbinding process, that on average first involves the escape from the hydrophobic binding pocket and subsequently from the surface region where residues conferring specificity are usually located. From the time history of all of the contacts that keep the peptide bound to the domain until they rupture, we obtained their average lifetimes, that correlate with a picture emerged form NMA analysis. This suggests a nontrivial coupling between collective dynamics of the molecule and binding mechanism even at physiological temperatures. This confims that normal mode analysis may provide a valuable method for detecting the protein chain fluctuations that represent the precursory events to unbinding.

Our results rest on the reliable calculation of the free energy profile of unbinding, which is a quantity defined at (quasi-)equilibrium, and on the precise determination of the escapetime histograms, which implies a complete description of the metastable state of the process, again a (quasi-)equilibrium quantity. This is consistent with the observation that experimental unbinding rates range from 1 to $10^{-3} \mathrm{~s}^{-1}$, time scales long enough for nanometric dynamical molecules such as PDZ domains to fully explore their phase space and consequently to be amenable to a (quasi-)equilibrium description.
Unfortunately, present all-atom MD with realistic force fields can access at most a few hundreds of nanoseconds of the dynamics, and are thus likely to miss events relevant on the second or longer time scales. Employing a native-centric backbone representation of the protein with simplified force field (Gō-like model) on the one hand allowed us a thorough investigation of the equilibrium and stationary properties of the system and on the other hand emphasized the role of the native state geometry in the binding mechanism of the PDZ3 domain. Given the overall high structural similarity of the PDZ family, our evidence for a diffusion-limited unbinding process likely applies to most if not all of them.

Although necessary to access the relevant time scales, neglecting the chemical details of the binding cleft and of the peptide comes at a price: it is impossible to precisely rank the affinities of different peptides for the same domain and of different domains for the same peptide. At the same time, the promiscuity of PDZ binding implies that some degree of generalized stickiness, captured by the simplified force field used here, is present. Moreover, we believe that our results represent a reading frame for future unbinding simulations that will become possible when algorithms and computational resources will be powerful enough to access the experimental time scales of the process while taking into account the full chemical detail.

At the same time, we stress that our prediction of diffusive versus thermally activated unbinding is surely amenable to experimental verification.

\section{References and Notes}

(1) Fanning, A. S.; Anderson, J. M. J. Clin. Invest. 1999, 103, 767772

(2) Ullmer, C.; Schmuck, K.; Figge, A.; Läbbert, H. FEBS Lett. 1998, 424, 63-68.

(3) Pawson, T.; Scott, J. D. Science 1997, 278, 2075-2080.

(4) Fan, J. S.; Zhang, M. Neurosignals 2002, 11, 315-321.

(5) Bezprozvanny, I.; Maximov, A. Proc. Natl. Acad. Sci. U.S.A. 2001, 98, 787-789.

(6) Kachinsky, A. M.; Froehner, S. C.; Milgram, S. L. J. Cell Biol. 1999, 145, 391-402.

(7) Faulkner, G.; Pallavicini, A.; Formentin, E.; Comelli, A.; Ievolella, C.; Trevisan, S.; Bortoletto, G.; Scannapieco, P.; Salamon, M.; Mouly, V.; Valle, G.; Lanfranchi, G. J. Cell Biol. 1999, 146, 465-475.

(8) Sheng, M.; Sala, C. Annu. Rev. Neurosci. 2001, 24, 1-29.

(9) Hung, A. Y.; Sheng, M. J. Biol. Chem. 2002, 277, 5699-5702.

(10) Doyle, D. A.; Lee, A.; Lewis, J.; Kim, E.; Sheng, M.; MacKinnon, R. Cell 1996, 85, 1067-1076.

(11) Au, Y.; Atkinson, A. R.; Guerrini, R.; Kelly, G.; Joseph, C.; Martin, S. R.; Muskett, F. W.; Pallavicini, A.; Faulkner, G.; Pastore, A. Structure 2004, 12, 611-622.

(12) Hillier, B. J.; Christopherson, K. S.; Prehoda, K. E.; Bredt, D. S.; Lim, W. A. Science 1999, 284, 812-815.

(13) Fujii, N.; Haresco, J. J.; Novak, K. A.; Kuntz, I. D.; Guy, R. K. Mol. Biol. Cell 2002, 13, 360A.

(14) Fujii, N.; Haresco, J. J.; Novak, K. A. P.; Stokoe, D.; Kuntz, I. D.; Guy, R. K. J. Am. Chem. Soc. 2003, 125, 12074-12075.

(15) Shan, J. F.; Shi, D. L.; Wang, J. M.; Zheng, J. Biochemistry 2005 44, 15495-15503.

(16) Saro, D.; Li, T.; Klosi, E.; Udugamasooriya, G.; Spaller, M. R. Biopolymers 2003, 71, 413-413.

(17) Wiedemann, U.; Boisguerin, P.; Leben, R.; Leitner, D.; Krause, G.; Moelling, K.; Volkmer-Engert, R.; Oschkinat, H. J. Mol. Biol. 2004, $343,703-718$.

(18) Udugamasooriya, G.; Saro, D.; Spaller, M. R. Org. Lett. 2005, 7, $1203-1206$

(19) Stiffler, M. A.; Grantcharova, V. P.; Sevecka, M.; MacBeath, G. J. Am. Chem. Soc. 2006, 128, 5913-5922.

(20) De Los Rios, P.; Cecconi, F.; Pretre, A.; Dietler, G.; Michielin, O.; Piazza, F.; Juanico, B. Biophys. J. 2005, 89, 14-21.

(21) Tama, F.; Sanejouand, Y. H. Protein Eng. Des. Sel. 2001, 14, 1-6. (22) Delarue, M.; Sanejouand, Y. H. J. Mol. Biol. 2002, 320, 10111024 . 
(23) Gerstein, M.; Krebs, W. Nucleic Acids Res. 1998, 26, 42804290.

(24) Madsen, K. L.; Beuming, T.; Niv, M. Y.; Chang, C. W.; Dev, K. K.; Weinstein, H.; Gether, U. J. Biol. Chem. 2005, 280, 20539-20548.

(25) Basdevant, N.; Weinstein, H.; Ceruso, M. J. Am. Chem. Soc. 2006 $128,12766-12777$.

(26) Gianni, S.; Engstrom, A.; Larsson, M.; Calosci, N.; Malatesta, F.; Eklund, L.; Ngang, C. C.; Travaglini-Allocatelli, C.; Jemth, P. J. Biol. Chem. 2005, 280, 34805-34812.

(27) Songyang, Z.; Fanning, A. S.; Fu, C.; Xu, J.; Marfatia, S. M.; Chishti, A. H.; Crompton, A.; Chan, A. C.; Anderson, J. M.; Cantley, L. C. Science 1997, 275, 73-77.

(28) Clementi, C.; Nymeyer, H.; Onuchic, J. J. Mol. Biol. 2000, 298, 937-953(17)

(29) Levy, Y.; Wolynes, P. G.; Onuchic, J. N. Proc. Natl. Acad. Sci. U.S.A. 2004, 101, 511-516.
(30) Cecconi, F.; Micheletti, C.; Carloni, P.; A., M. Proteins: Struct., Funct., Genet. 2001, 43.

(31) Gianni, S.; Calosci, N.; Aelen, J. M.; Vuister, G. W.; Brunori, M.; Travaglini-Allocatelli, C. Protein Eng. Des. Sel. 2005, 18, 389-395.

(32) Morriss, G. P.; Dettmann, C. P. CHAOS 1998, 8, 321-336.

(33) Ferrenberg, A. M.; Swendsen, R. H. Phys. Rev. Lett. 1988, 61, $2635-2638$

(34) Valleau, J. P.; Card, D. N. J. Chem. Phys. 1972, 57, 5457-5462.

(35) Gardiner, C. W. Handbook of Stochastic Methods; SpringerVerlag: Berlin, 1985.

(36) Mel'nikov, V. I.; Meshkov, S. V. J. Chem. Phys. 1986, 85, 10181027.

(37) Klimov, D. K.; Thirumalai, D. Phys. Rev. Lett. 1997, 79, 317320.

(38) Lockless, S. W.; Ranganathan, R. Science 1999, 286, 295-299.

(39) Dima, R. I.; Thirumalai, D. Protein Sci. 2006, 15, 258-268. 